\title{
Multi-Agent Search for Source Localization in a Turbulent Medium
}

\author{
Hadi Hajieghrary ${ }^{a}$, M. Ani Hsieh ${ }^{a}$, and Ira B. Schwartz ${ }^{b}$ \\ ${ }^{a}$ Scalable Autonomous Systems Lab, Mechanical Engineering \& Mechanics, Drexel \\ University, Philadelphia, PA 19104, USA \\ ${ }^{b}$ Nonlinear Systems Dynamics Section, Plasma Physics Division, Code 6792, U.S. Naval \\ Research Laboratory, Washington, DC 20375, USA
}

\begin{abstract}
We extend the gradient-less search strategy referred as "infotaxis" to a distributed multi-agent system. "Infotaxis" is a search strategy that uses sporadic sensor measurements to determine the source location of materials dispersed in a turbulent medium. In this work, we leverage the spatio-temporal sensing capabilities of a mobile sensing agents to optimize the time spent finding and localizing the position of the source using a multi-agent collaborative search strategy. Our results suggest that the proposed multi-agent collaborative search strategy leverages the team's ability to obtain simultaneous measurements at different locations to speed up the search process. We present a multi-agent collaborative "infotaxis" strategy that uses the relative entropy of the system to synthesize a suitable search strategy for the team. The result is a collaborative information theoretic search strategy that results in control actions that maximize the information gained by the team, and improves estimates of the source position.
\end{abstract}

Keywords: multi-agent systems, information theory, distributed control

\section{Introduction}

Nature has been optimizing search strategies in complex uncertain environments for billions of years. For example, the efficiency of male moths searching for females is quite remarkable. In spite of a slew of serious obstructions in signal detection and processing, the olfactory pheromone system usually guarantees a successful encounter [1]. Far from the pheromone emitting female, odor plumes consist of sparsely distributed pheromone patches 
due to turbulence [2], leading to rare, intermittent detections [3, 4]. These and similar search behaviors in biological systems give insight into the mechanisms linking perception to action, and for developing more effective search strategies in general. The understanding of how ants and honeybees locate and return to their respective colonies when foraging for food has led to new probabilistic graph search strategies like ant and bee colony optimization $[5,6]$. These successes have further increased interest in developing similar strategies for autonomous mobile robots for search and rescue applications like the detection of gas leaks and exploration of buildings on fire [7], tracking of hazardous chemical plumes [8], and multi-robot exploration of unknown environments $[9,10]$.

Existing strategies for detecting, tracking, and localizing gas/odor/radiation sources with mobile robots include building of flow field maps [11, 12, 13], estimating concentration gradients $[14,15,16,17,18,19]$, and using gradientfree search algorithms $[16,17]$. However, the variation in chemical concentrations from a source in a flow environment is heavily dependent on the Reynolds numbers. In general, gradient-based approaches generally work better in lower Reynolds regimes since the variation in chemical concentrations are generally smoother [20]. However, as the Reynolds number increases, dispersion of the chemical source becomes increasingly dominated by turbulent mixing which renders many gradient-based strategies impractical $[21,22,23,24,25]$.

To address some of these challenges, various bio-inspired strategies based on bacteria [15, 16, 17], insects [26, 27, 28], and crabs [29] have also been proposed. However, these bio-inspired approaches are mostly ad-hoc, focused on developing novel sensor technology [30, 27], or are equivalent to coverage and gradient based search strategies for single robots $[16,17,26,27,28,20]$. Alternatives to these existing strategies include a new class of reactive search strategies. These strategies do not rely on continuous or smooth concentration gradients and can adapt to past sensory information and action $[4,11,31]$. The so called "infotaxis" strategy maximizes the information gain about the location of the source in a turbulent medium [4]. While these strategies are more sophisticated, they are also computationally expensive and have almost exclusively focused on adaptive behavior in the context of single agent search strategies.

The main contribution of this work is the extension of the single-agent information theoretic search strategy coined as "infotaxis" to a multi-agent robotic system [4]. As such, we present two collaborative information maxi- 
mizing search strategies for the multi-robot team and compare their performances to the single agent "infotaxis" strategy. The paper is organized as follows: We briefly summarize the single agent "infotaxis" strategy and lay out our assumptions in Section 2. We present the multi-agent "infotaxis" search strategy in Section 3.1 and present our simulation results in Section 4. The comparison and discussion of the single- and multi-agent strategies is presented in Section 5. We conclude with a summary of our results in Section 6.

\section{Background}

The main objective is to extend the single-agent "infotaxis" search strategy presented in [4] to a multi-robot system. We begin by outlining our assumptions and briefly summarizing the single-agent strategy.

\subsection{Assumptions}

Given an obstacle free workspace in two dimensions (2D) denoted by $\mathcal{W}$, we assume $\mathcal{W}$ is discretized into uniform grid cells. A cell is occupied if the agent, or robot, is located within it and agents are only allowed to move from their current cell to any of the eight adjacent cells, i.e., up, down, left, right, and diagonally across the four corners. As such, each agent is effectively modeled as a single massless point particle with omnidirectional kinematics. Each robot is equipped with a binary chemical sensor that is capable to detect the presence (or absence) of chemical at the agent's current location in $\mathcal{W}$. We also assume every agent has the ability to localize within $\mathcal{W}$, i.e., know its position, and can measure the magnitude and direction of the local flow field at its current location. Lastly, we assume each agent can communicate with every other member of the team. While inter-agent information exchange can be done asynchronously, we assume no delays in communication since the focus of this work is to compare the performance of the multi-agent strategy with the single-agent system rather than to study the effects of communication delays on the search strategy.

In general, the expected rate of positive material plume detection in an environment depends on the spatial distance to the source, the dynamics of the surrounding flow field, the geometry of the environment, and many other factors. Due to the complexity of the dispersion dynamics of biochemical and/or radiological material in a turbulent medium, similar to [4], we model the rate of detecting the presence of a material plume as a Poisson 
distribution. The statistical model for positive material plume detection in a turbulent medium given in [4] is briefly summarized below.

The mean rate of positive detection at position $\mathbf{r}$ for a source located at $\mathbf{r}_{\mathbf{0}}$ in $2 \mathrm{D}$ is given by:

$$
\begin{aligned}
R\left(\mathbf{r} \mid \mathbf{r}_{\mathbf{0}}\right) & =\frac{\mathcal{R}}{\ln \left(\frac{\lambda}{a}\right)} e^{\frac{\left(y_{0}-y\right) V}{2 D}} K_{0}\left(\frac{\left|\mathbf{r}-\mathbf{r}_{\mathbf{0}}\right|}{\lambda}\right), \text { with } \\
\lambda & =\sqrt{\frac{D \tau}{1+\left(\frac{V^{2} \tau}{4 D}\right)}},
\end{aligned}
$$

where $\mathcal{R}$ is the emission rate of the source, $\tau$ is the finite lifetime of the chemical patch before its concentration falls off the detectable range, $D$ is the isotropic effective diffusivity of the medium, and $V$ is the mean velocity of the wind, and $K_{0}(\cdot)$ is the modified Bessel function of the second kind. Similar to [4], we assume a strong background directional flow that is predominantly in the $-y$ direction.

Let $P_{t}\left(\mathbf{r}_{\mathbf{0}}\right)$ denote the estimated probability distribution that describes the possible locations of the source in $\mathcal{W}$. This probability distribution function at time $t$ represents the information gathered through a series of uncorrelated positive sensor measurements or positive odor encounters. In general, $P_{t}\left(\mathbf{r}_{\mathbf{0}}\right)$ can be computed using Bayes' rule:

$$
P_{t}\left(\mathbf{r}_{\mathbf{0}}\right)=\frac{P_{\mathbf{r}_{\mathbf{0}}}\left(\mathbf{z}_{1: t}\right)}{\int_{\mathcal{W}} P_{\mathbf{r}}\left(\mathbf{z}_{1: t}\right) \mathrm{d} \mathbf{r}},
$$

where $\mathbf{r}$ denotes a position in the workspace $\mathcal{W}, \mathbf{z}_{1: t}$ denotes the history of odor encounters, and $P_{\mathbf{r}_{0}}\left(\mathbf{z}_{1: t}\right)$ is the likelihood of obtaining such a history of sensor measurements if the source is located at $\mathbf{r}_{\mathbf{0}}$. The expected rate of positive sensor measurements at any given location in $\mathcal{W}$ is a function of the relative position of the agent with respect to the source. Assuming that the detection of the plume at every location in $\mathcal{W}$ is independent of its neighboring positions, we use Poisson's law to estimate the number of detections at each step during the exploration as in [4]. We note that the assumption of independence for the detection probability holds since the location of the source is unknown. As such, $P_{\mathbf{r}_{\mathbf{0}}}\left(\mathbf{z}_{1: t}\right)$ is given by an exponential distribution of the form

$$
P_{\mathbf{r}_{\mathbf{0}}}\left(\mathbf{z}_{1: t}\right)=\exp \left(\left[-\int_{0}^{t} R\left(\mathbf{r}\left(t^{\prime}\right) \mid \mathbf{r}_{\mathbf{0}}\right) \mathrm{d} t^{\prime}\right] \prod_{i=1}^{h} R\left(\mathbf{r}\left(t_{i}\right) \mid \mathbf{r}_{\mathbf{0}}\right)\right)
$$


where $\mathbf{r}(t)$ denotes the positions where the agent obtained positive sensor readings, i.e., detection of the presence of the chemical/plume, and $h$ is the number of such detections.

\subsection{Single-Agent Search Strategy}

To maximize the expected rate of information gain for the source location, the single-agent search strategy in [4] is designed to maximize the expected reduction of the entropy of $P_{t}(\mathbf{r})$. Since the entropy of $P_{t}(\mathbf{r})$ is defined as $S_{t}=-\int_{\mathcal{W}} P_{t}(\mathbf{r}) \log \left(P_{t}(\mathbf{r})\right) \mathrm{d} \mathbf{r}$, the expected change in entropy for an agent moving from its current location $\mathbf{r}$ to another location $\mathbf{r}_{j}$ in $\mathcal{W}$ at current time step $t$ is given by:

$$
\mathbf{E} \llbracket \Delta S_{t}\left(\mathbf{r} \mapsto \mathbf{r}_{j}\right) \rrbracket=P_{t}\left(\mathbf{r}_{j}\right)\left[-S_{t}\right]+\left[1-P_{t}\left(\mathbf{r}_{j}\right)\right]\left[\left(1-\rho\left(\mathbf{r}_{j}\right)\right) \Delta S_{0}+\rho\left(\mathbf{r}_{j}\right) \Delta S_{1}\right] .
$$

The first term in (4) corresponds to the change in entropy should the agent find the source at the next step. The second term of (4) accounts for the likelihood that the source is not at $r_{j}$ and calculates the mean value of the information gained from additional encounters. Under these circumstances, we consider two possible outcomes: the agent obtains a positive or negative sensor measurement at the new position. In (4), $\rho\left(\mathbf{r}_{j}\right)$ denotes the probability of detection made at the next step. The expected number of positive measurements at the new position is calculated based on the current estimate of $P_{t}(\mathbf{r})$. Here, the observation model is the expected rate of positive sensor measurements at position $\mathbf{r}$ if the source is located at $\mathbf{r}_{\mathbf{0}}$ given by $R\left(\mathbf{r}(t) \mid \mathbf{r}_{\mathbf{0}}\right)$. Given the estimate of the source location, the expected number of hits at any location in the workspace is given by:

$$
h\left(\mathbf{r}_{j}\right)=\int P_{t}\left(\mathbf{r}_{j}\right) R\left(\mathbf{r}_{j} \mid \mathbf{r}_{\mathbf{0}}\right) \mathrm{d} \mathbf{r}_{\mathbf{0}}
$$

where the probability of a single positive detection follows the Poisson law $\rho\left(\mathbf{r}_{j}\right)=h\left(\mathbf{r}_{i}\right) \exp \left(-h\left(\mathbf{r}_{j}\right)\right)$. In (4), $\Delta S_{0}$ denotes the entropy change of the probability density function of the source location if the agent receives no new positive sensor measurements at the next time step as it moves to the neighboring cell. The change in entropy of the probability density function if a detection is made at the new position is given by $\Delta S_{1}$. The result is a search strategy that favors agent motions that maximize the likelihood of finding the source location. We refer the interested reader to [4] for the complete details. 


\section{Collaborative Multi-Agent Search}

The main main objective of this work is to build upon the original work of [4] and develop a multi-agent collaborative gradient-free search strategy that locally maximizes the rate of information gain at every step. In general, the more information one can gather the more exact the estimate for the source position. Given the sporadic nature of the plume detections by any given agent, it makes sense to employ a group of agents to more efficiently explore the workspace to localize the source's position. In fact, "infotaxis" as a search strategy can be easily formulated as a multi-agent search strategy since information gathered by each agent along its trajectory can be shared with other agents. Given a team of $N$ mobile sensing agents, we present two collaborative multi-agent "infotaxis" derived strategies: the Simple Collaborative Search and the KL-Based Collaborative Search.

\subsection{Simple Collaborative Search}

For the simple collaborative search strategy, we assume each agent in the team individually executes the single agent "infotaxis" search strategy described in Section 2.2. In this strategy, every agent $i$ maintains an estimate of the probability distribution that describes the possible source location which we denote as $P_{t}^{i}(\mathbf{r})$. Whenever agent $i$ detects the presence of the material plume, it communicates its coordinates to the rest of the team. Every agent in the team, i.e., $j \in\{1, \ldots, N\}$ such that $j \neq i$, then updates its own likelihood function with the information provided by the reporting agent $i$. In the absence of communication delays or loss of information, the likelihood function for the source location is identical among all agents in the team, i.e., $P_{t}(\mathbf{r})=P_{t}^{1}(\mathbf{r})=\ldots=P_{t}^{N}(\mathbf{r})$. We note that this strategy naturally allows for asynchronous updates to each agent's estimate of $P_{t}(\mathbf{r})$. To determine their next move, each agent uses their own estimates of the density function, $P_{t}^{i}(\mathbf{r})$, to determine the next location to move to and predict the next locations the other agents in the team will move to.

By increasing the number of agents searching for the source location, one would expect a decrease in the time needed to find the source and a decrease in the variance of the estimate of the source position. This is because the additional number of agents effectively increases the detection rate of the team. As such, for a team of $N$ mobile sensing agents, the team would explore less of the workspace, resulting in less time needed to find and estimate

the source position. However, a potential downside is the detection of false 
positives since the team may decide to stop the search once the variance of the source position estimate drops below a pre-specified threshold.

\subsection{KL-Based Collaborative Search}

In general, false positives for the source position can be difficult to reject, especially in the absence of complementary sensor data. To minimize the chances for false positives, we present the KL-based collaborative search strategy for a team of $N$ mobile sensing agents. Similar to the simple collaborative search strategy, the KL-based strategy will also be designed to maximize the information gained by the team. Different from the simple collaborative search strategy, the KL-based strategy will rely on the KullbackLeibler (KL) divergence, i.e., the relative entropy, rather than the absolute entropy of $P_{t}(\mathbf{r})$.

For every agent $i$, we assume it obtains its own estimate of the density function describing the possible source locations without incorporating information from other agents in the team which we denote $P_{t}^{i}(\mathbf{r})$. Instead of relying on the entropy of the team's source position likelihood density function, $P_{t}(\mathbf{r})$, each agent uses the KL divergence to measure the difference between its source position probability density function and the density functions of the other team members [32]. The KL divergence or relative entropy between two likelihood functions $P_{t}^{i}(\mathbf{r})$ and $P_{t}^{j}(\mathbf{r})$ is given by:

$$
D\left(P^{i} \| P^{j}\right)=\sum_{r \in \mathcal{W}} P^{i}(\mathbf{r}) \log \frac{P^{i}(\mathbf{r})}{P^{j}(\mathbf{r})} .
$$

The KL divergence, $D\left(P^{i} \| P^{j}\right)$, is a measure of the error in assuming that some random variable is drawn from a distribution $P^{j}$ when the true distribution is in fact $P^{i}$.

In this strategy, each agent estimates the probability distribution of the source positive based on its own sensor measurements. To increase the confidence of each agent's estimates, each agent derives its control action such that the relative entropy of the likelihood function for the source position currently estimated by the other agent, i.e., $P_{t}^{j}(\mathbf{r})$, and the expected likelihood function of the source upon agent $i$ 's next move, $D\left(P_{t}^{j} \| \mathbf{E} \llbracket P_{t+1}^{i} \rrbracket\right)$, is minimized. The expected probability function for the source position upon agent $i$ 's next move is given by

$$
\mathbf{E} \llbracket P_{t+1}^{i} \rrbracket=P_{t}^{i}\left(\mathbf{r}_{k}\right)+\left[1-P_{t}^{i}\left(\mathbf{r}_{k}\right)\right]\left[\left(1-\rho\left(\mathbf{r}_{k}\right)\right) S_{t+1}(X=0)+\rho\left(r_{k}\right) S_{t+1}(X=1)\right]
$$


where $P_{t+1}^{i}$ denotes agent $i$ 's likelihood function of the source position at the next step. Additionally, $S_{t+1}(X=0)$ and $S_{t+1}(X=1)$ denote the probability of a negative and positive sensor measurement, respectively, at the very next step which are calculated based on the current estimate of $P_{t}^{i}$.

Equation (7) computes the expected likelihood function based on the expected number of positive sensor measurements at any of the robot's neighboring positions/cells. The result is a strategy where every agent tries to minimize the error of its estimate of the source position by comparing it with the other agent's estimates. Different from the simple collaborative search strategy, the proposed strategy should enable the team to better exploit the information gathered by the various agents.

\section{Simulation Results}

In this section we present the simulation results for the single- and multiagent "infotaxis" search strategies. The single-agent "infotaxis" strategy serves as the baseline for comparison.

\subsection{Single Agent Search}

In the absence of positive sensor measurements, the proposed information theoretic search strategy favors random exploration of the workspace over actively seeking the source. However, when an agent obtains a positive sensor measurement, the new information reshapes the likelihood estimate of the source location. The updated probability density function guides the agent to the source along a mostly straight path. This can be seen in Fig. 1 and Fig. 2 where the likelihood functions for the estimated source position by a single agent executing the "infotaxis" strategy and the agent's trajectories are shown. Fig. 1 and 2 show the simulation results for the single agent in an environment without and with an external directional flow respectively.

We note that in the presence of an external directional flow, the agent achieves a good estimate of the possible target position in $\mathcal{W}$ after 200 time steps (see Fig. 2). After 300 time steps, the estimate is good enough for the agent to successfully localize the source with an acceptable level of confidence. In the presence of an external directional flow, the first positive sensor measurement provides a great deal of information about the location of the source. This is especially true when agents have the ability to measure or estimate the direction of the background flow, since it allows the agent to limit its search to the areas upwind. The result is a dramatic initial change in the 


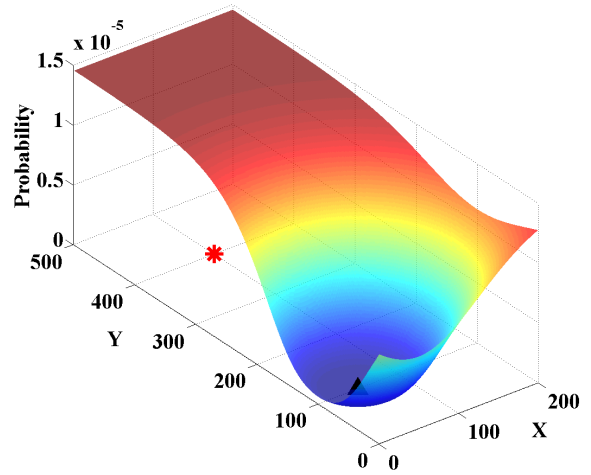

(a) Time Step 0

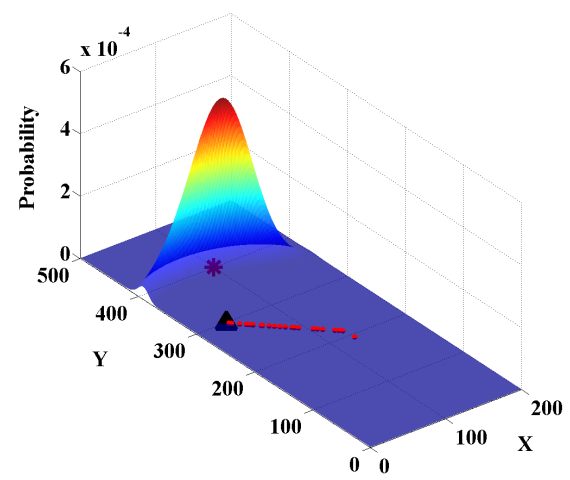

(c) Time Step 200

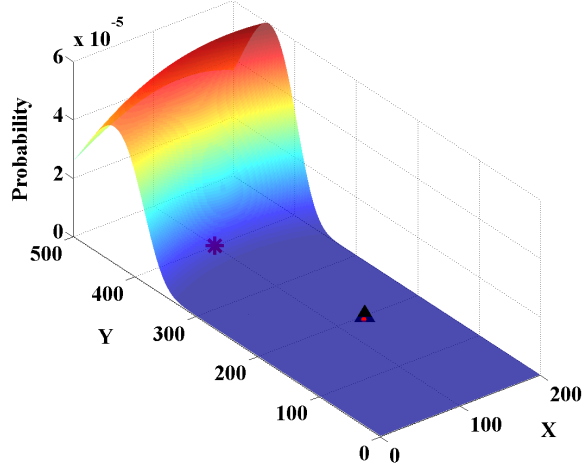

(b) Time Step 100

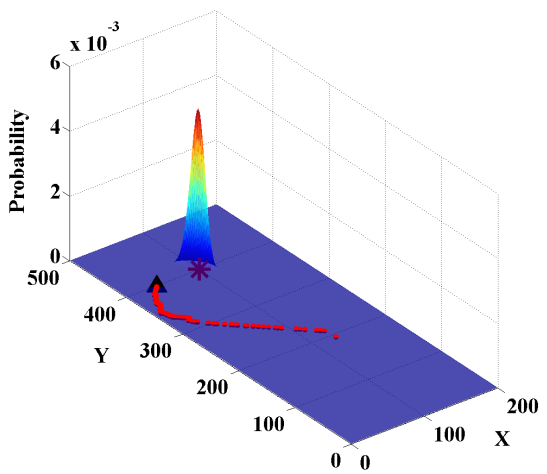

(d) Time Step 300

Figure 1: Estimated likelihood function for the source position in an environment without any external flows at time steps $0,100,200$, and 300. In this simulation $D=1, \tau=2500$, $R=100$, and $a=1 e-4$. The asterisk denotes the source located at $(100,400)$. The black triangle denotes the agent's current position. The red dots indicate the locations where the agent obtained a positive sensor measurement. 


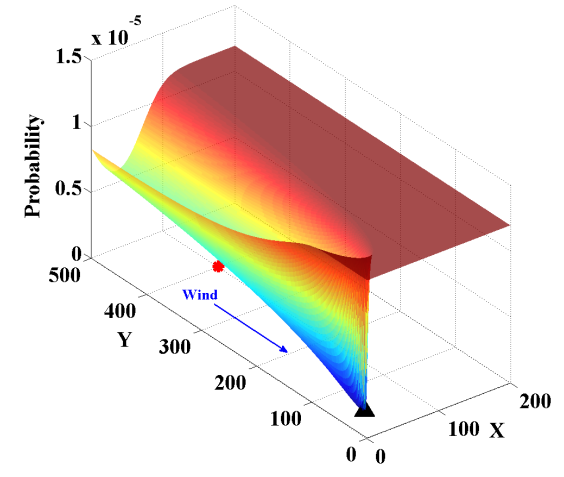

(a) Time Step 0

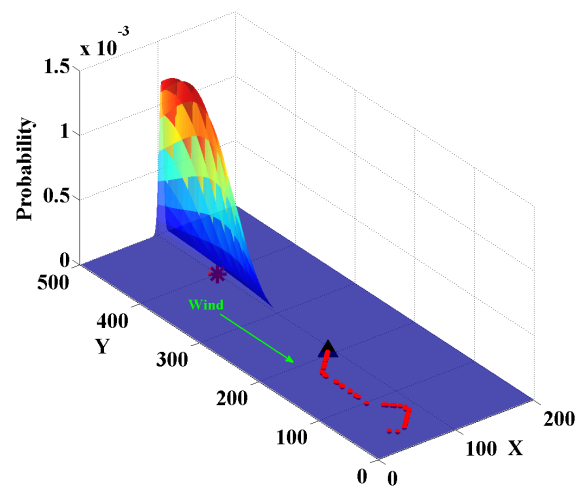

(c) Time Step 200

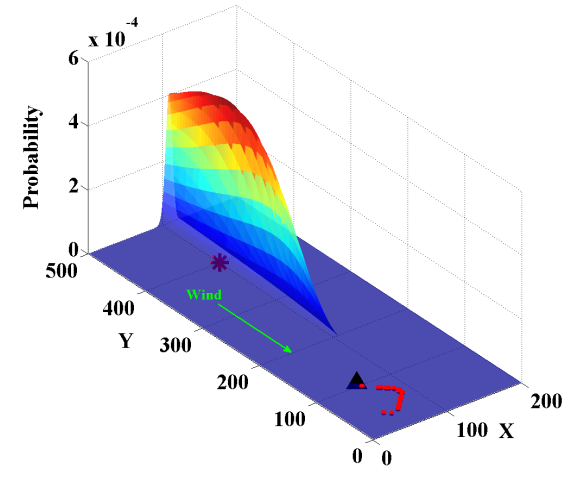

(b) Time Step 100

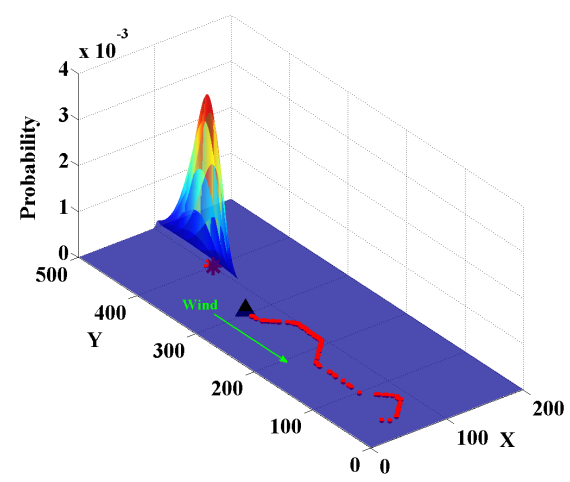

(d) Time Step 300

Figure 2: Estimated likelihood function for the source position in an environment with a directional external flow at time steps $0,100,200$, and 300. In this simulation $D=1$, $\tau=2500, R=100$, and $a=1 e-4$. The mean magnitude of the external flow velocity is $V=1$ units per second and the direction is denoted by the arrow. The asterisk denotes the source located at $(100,400)$. The black triangle denotes the agent's current position. The red dots indicate the locations where the agent obtained a positive sensor measurement. 


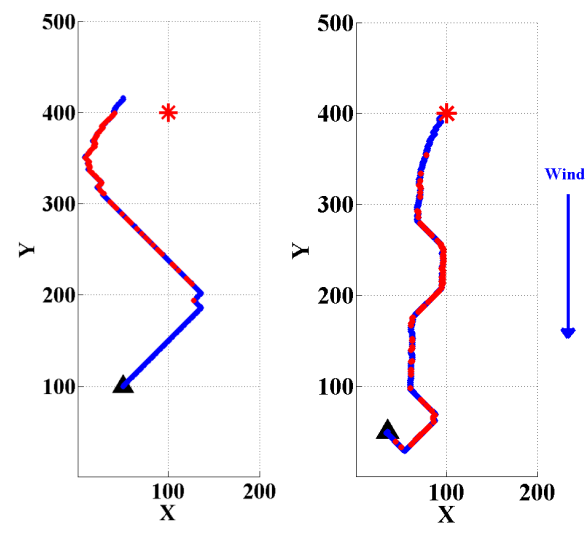

(a)

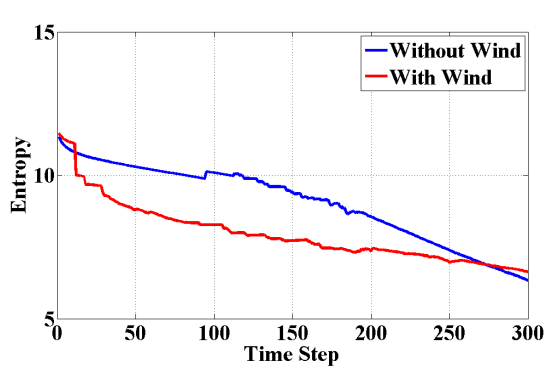

(b)

Figure 3: (a) Sample trajectory of the agent in the environment without (left) and with (right) the external directional flow. The red dots denote locations where the agent received positive sensor measurements. The black triangle denotes the initial position of the agent and the asterisk denotes the location location of the source. (b) The entropy of the estimated likelihood function for the source position over time without and with the external background flow.

estimated probability distribution function describing the source location. However, the rate of information acquisition by the agent would also drop dramatically given that subsequent positive measurements contain much less information as compared to the first positive measurement. This is shown in Fig. 3b where the entropy of the probability distribution estimated at each time step by the single agent is shown over time. Since positive sensor measurements are random, the information gathered in the absence of an external directional flow decreases linearly over time. In contrast, the presence of the directional flow results in a faster decrease in the entropy of the estimated probability distribution.

\subsection{Simple Collaborative Search}

In contrast to the single-agent strategy, Fig. 4, 5, and 6 show the simulation results for a group of three agents looking for the plume source in an obstacle free environment using the Simple Collaborative Search strategy described in Section 3.1 in an environment without and with an external unidirectional flow. We note that in the presence of an external unidirectional flow, the is able to arrive at a more precise initial estimate of the source location (see Fig. 5a vs. 6a). 

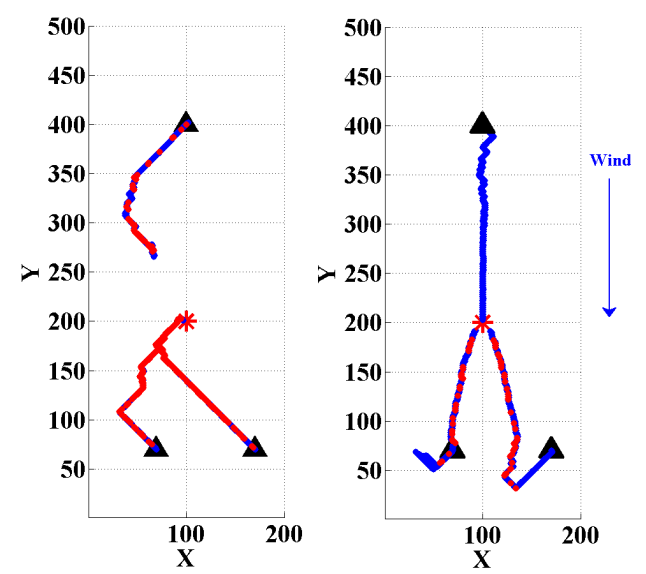

Figure 4: Trajectories of the team's in the environment without (left) and with (right) the external directional flow. The red dots denote locations where the agent received positive sensor measurements. The black triangle denotes the initial position of the agent and the asterisk denotes the location location of the source. In these simulations $D=1, \tau=2500$, $R=100$, and $a=1 e-4$. The asterisk denotes the source located at $(100,200)$.

Since computation of the expected change in entropy can be costly, these simulations were performed such that each agent determines its control actions by only taking into account the change in entropy resulting from its own action, rather than the actions of the entire team. However, since each agent maintains an estimate of the likelihood function for the source location and updates their estimates based on the positive sensor measurements obtained by every member in the team, the strategy remains a collaborative one. We note that while this implementation results in variations between each agent's belief distribution of the source position, variations are small and mostly negligible.

\subsection{KL-Based Collaborative Search}

Fig. 7 shows the likelihood density function describing the source position estimated by one agent performing the KL-based collaborative search strategy for a team of two agents. The multi-modal nature of the density function results from the fact that each agent's control strategy only relies on each agent's estimate of the likelihood function describing the source position. In the KL-based strategy, individual agents do not fuse information from the other agents into their estimate of the source position. Rather, the positive 


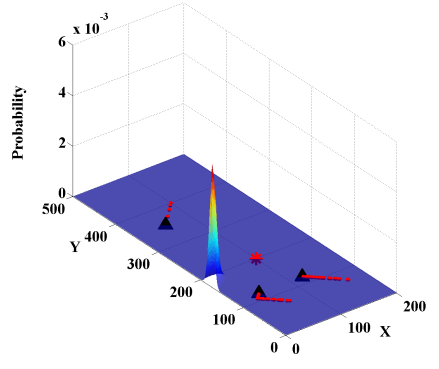

(a) Time step 50

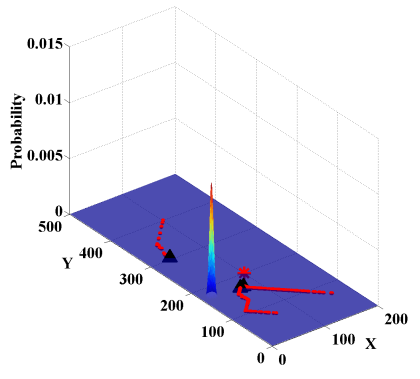

(b) Time step 100

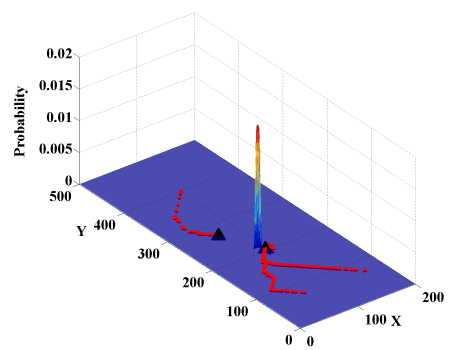

(c) Time step 140

Figure 5: Estimated likelihood function for the source position in an environment without an external flow at time steps 50, 140, and 250. In this simulation $D=1, \tau=2500$, $R=100$, and $a=1 e-4$. The asterisk denotes the source located at $(100,200)$. The black triangle denotes the agent's current position. The red dots indicate the locations where the agent obtained a positive sensor measurement.

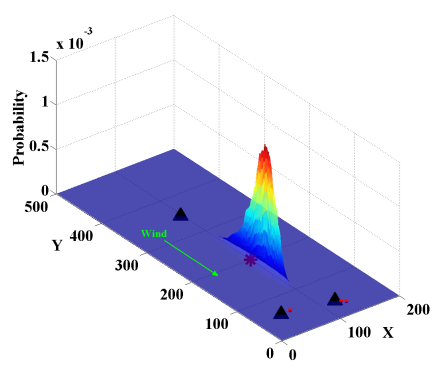

(a) Time step 50

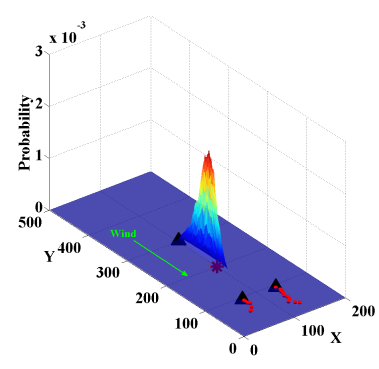

(b) Time step 100

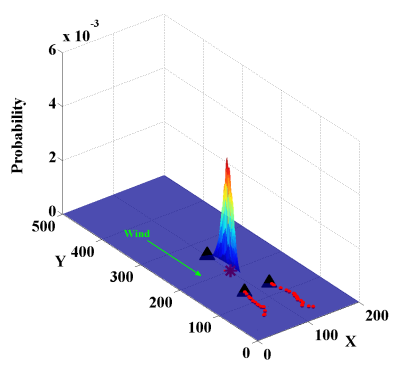

(c) Time step 140

Figure 6: Estimated likelihood function for the source position in an environment with a directional external flow at time steps 50; 140; and 250. In this simulation $D=1$, $\tau=2500, R=100$, and $a=1 e-4$. The mean magnitude of the external flow velocity is $V=1$ units per second and the direction is denoted by the arrow. The asterisk denotes the source located at $(100,200)$. The black triangle denotes the agent's current position. The red dots indicate the locations where the agent obtained a positive sensor measurement.

detections, or information, from the other agents are only used to determine the agent's next move. As such, the resulting search strategy is one where control actions by individual agents minimizes the difference between the two agents' likelihood functions.

Fig. 8a shows the entropy of the estimated probability distribution for the location of the source as determined by Agent 1. Since individual agents do not fuse the sensory information provided by other agents in the team, 


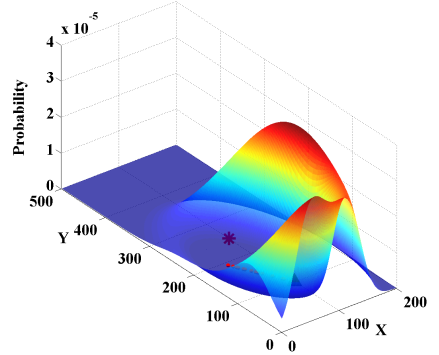

(a) Agent 1: Time Step 100

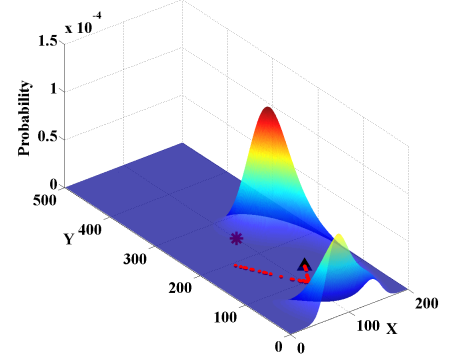

(b) Agent 1: Time Step 150

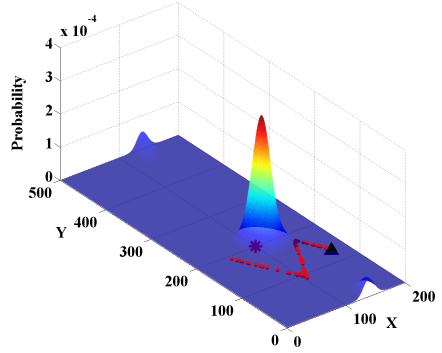

(c) Agent 1: Time Step 200

Figure 7: Estimated likelihood function for the source position by Agent 1 in an environment without an external flow at time steps 100, 200, and 250. In this simulation $D=1$, $\tau=2500, R=100$, and $a=1 e-4$. The asterisk denotes the source located at $(100,200)$. The black triangle denotes the agent's current position. The red dots indicate the locations where the agent obtained a positive sensor measurement.

the resulting decrease in entropy is driven by the control actions performed by each agent. From Fig. 8a we see that the proposed KL-based strategy successfully decrease the time needed by the team to localize the source without resulting in erroneous estimation of the source position.

\section{Discussion}

As expected, increasing the number of agents effectively decreases the time needed to localize the position of the source. This is shown in Fig. 8b where the entropy over time decreases exponentially faster for a multi-agent team as compared to a single agent. In the case when the source is located in an environment exhibiting a constant directional flow, agents positioned upwind of the source would not obtain any positive measurements. Under these circumstances, agents that are upwind would simply move directly towards the estimated source position using information gleaned by other agents located downwind from the source. In such a configuration, we note differentiation in the agents behaviors. Agents located downwind from the source tend to focus on exploring the workspace while agents located upwind exploits the information provided by the downwind agents to seek out the source. This phenomena can be seen in Fig. 4 where the trajectory of the agent placed upwind of the source in an environment with a directional flow consists of a straight line to the estimated source position. 


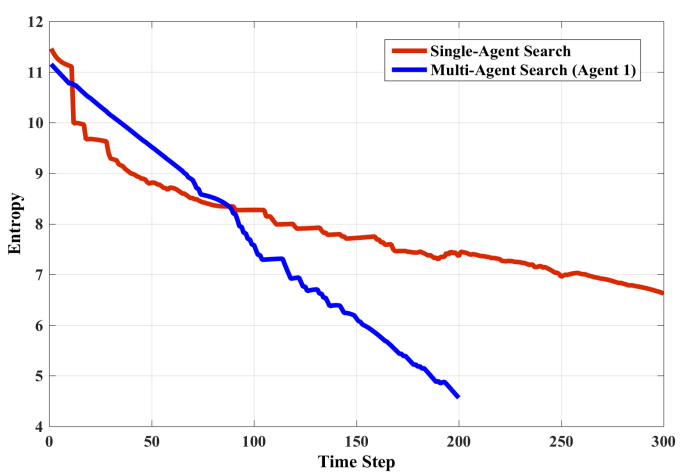

(a)

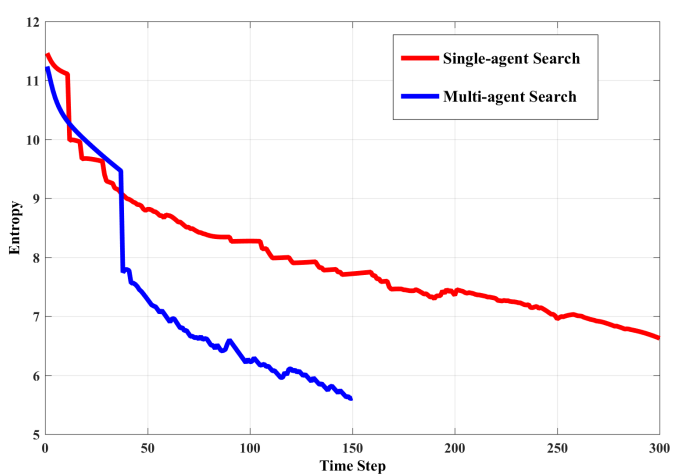

(b)

Figure 8: (a) Comparison of the entropy of the likelihood function of the source position for the single agent search strategy described in Section 2 and the entropy of the likelihood function of the source position as computed by one agent using the described KL divergence based strategy without an external unidirectional flow. (b) The entropy of the estimated likelihood function describing the source position as obtained by the single robot and by the team for the scenarios shown in Fig. 1 and the left panel of Fig. 4, i.e., without wind.

Furthermore, our results also show a significant reduction in the variance of the estimate of the source position when employing multiple agents versus a single agent as shown in Fig. 9. This is because the added agents effectively increases the detection rate of the team as a whole, making the team more confident in its estimate of the source position. However, this reduction in uncertainty in the source position estimate can potentially lead the team to an incorrect estimate of the source location as shown in Fig. 4 where the robots collectively move towards the left side of the workspace. This is the equivalent to obtaining a false positive detection for the source position. Fortunately, this erroneous estimate is eventually corrected since they eventually turn back towards the source. As agents move closer to the erroneous position estimate, the lack of positive signal cues coming from the erroneous position in combination with the positive detections originating a different position estimate eventually drives the team to the correct source position estimate.

To better understand how the multi-agent collaborative infotaxis search strategy can lead to erroneous estimates of the source position, we compare the results for the single and multi-agent system in the absence of a background directional flow. Fig. 8b shows the entropy of the position likelihood function for both the single and multi-agent cases. The entropy of the multi- 


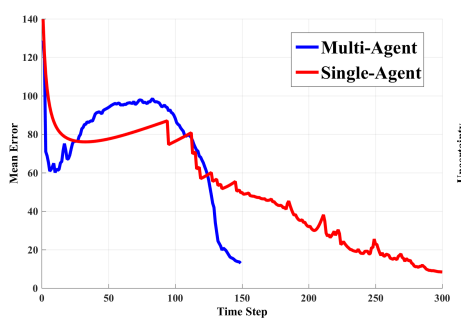

(a) Mean Error

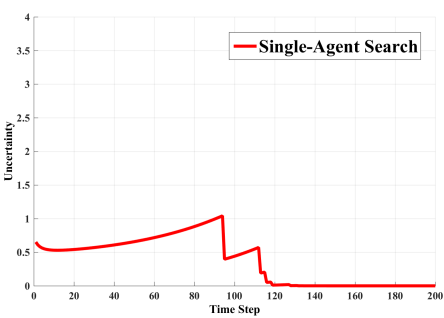

(b) Single Agent

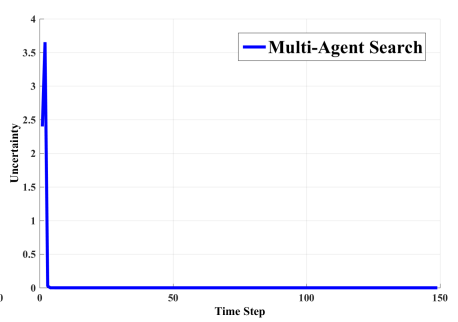

(c) Multi-Agent

Figure 9: (a) Mean error of the source position estimate for the single and multi-agent systems. (b) Uncertainty of the single agent estimate of the source position as measured by the norm of the covariance of the source position likelihood function. (c) Uncertainty of the single agent estimate of the source position as measured by the norm of the covariance of the source position likelihood function. All panels show data from simulations with an external unidirectional flow.

agent system decreases more sharply when compared to the single agent case. This is not surprising since multiple agents can gather more information than a single one. However, since the detection of the plume at every agent's position is sporadic, one would expect to see temporary increases in the estimation error of the source position over the duration of a search. This is particularly true for the single agent case when it is focused on exploring the workspace rather than actively seeking the source as shown in Fig. 9a. As the single agent explores the workspace, this behavior results in a temporary increase in the estimation error of the source position. This temporary increase in the single agent's estimation error results in a corresponding decrease in its confidence (or increase in uncertainty) of its own estimate of the position of the source as shown in Fig. 9b. In contrast, in the multi-agent case, an increase in the estimation error does not result in a decrease in the team's confidence of its estimate of the source position. In fact, as Fig. 9c shows, the team's confidence in its estimate of the source position remains high (or the uncertainty remains low) throughout the entire search.

In the multi-agent case, such erroneous estimates are only corrected when the agents move close enough to the estimated location to verify whether the source is present or not. This is seen in the trajectories of the mobile sensing agents in Fig. 4 where the team eventually turns away from the incorrect position estimate and eventually converges to the correct source position estimate. Had the team ended its search when the uncertainty of the position estimation dropped below some given threshold, the team 
would have reported an incorrect estimate for the source position. As such, we conclude that the simple collaborative search strategy results in team behaviors that forgoes exploration of the workspace in favor of active source seeking. However, this trade-off can lead to potential false positives which results in incorrectly estimating the source position.

Fortunately, the KL-based collaborative search strategy successfully retains the advantages of the multi-agent search without sacrificing estimation quality. The result is a much more successful cooperative search strategy compared to the simple collaborative search strategy. While the proposed KL-based collaborative search strategy is focused on teams of two agents, it can easily be extended to any team of $N>2$ agents. When $N>2$, each agent would then determine the next move by minimizing the joint relative entropy between its estimate of the likelihood function for the source location and that of the other team members. It is important note that the KL-based strategy may result in an agent visiting the same cell in the workspace twice. While this may result in search inefficiencies, the proposed strategy does result in better guiding of the agents to regions in the workspace that maximizes the changes of receiving positive sensor measurements and information flow.

\section{Conclusion}

We have presented a comparative study of the single agent information theoretic search strategy, often referred as "infotaxis", with two collaborative multi-agent "infotaxis" inspired search strategies. "Infotaxis" is a strategy where agents maximize the change in entropy of the likelihood function estimate of the position of a plume source [4]. The strategy is a gradient-less search strategy that has been shown to be robust even in turbulent and highly stochastic mediums. In this work we showed that the multi-agent collaborative "infotaxis" speeds up the search process by leveraging the team's ability to obtain simultaneous measurements at different locations in the workspace. However, we also show a simple extension of the single agent "infotaxis" search strategy can lead to over confidence in the team's estimates resulting in incorrect estimates of the source position. This is because in the multi-agent case, the entropy minimizing strategy overly favors source seeking to the extent where the team spends too little time exploring the workspace. Based on our analysis, we presented a variant of the multi-agent collaborative search strategy where the KL divergence or relative entropy of 
the system was used to derive an appropriate control strategy for the team. The result is a collaborative information theoretic search strategy that results in search strategies that better maximizes the information gained by the team, leading to more accurate estimates of the source position.

\section{Acknowledgements}

HH and MAH were supported by ONR N000141211019 and Gulf of Mexico Research Initiative via Consortium for Ocean Leadership, Inc. Award No. SA 15-15. IBS was supported by ONR grant N0001414WX20610 and NRL based funding N0001414WX00023.

[1] R. T. Carde, M. A. Willis, Navigational strategies used by insects to find distant, wind-borne sources of odor, Journal of Chemical Ecology 34 (7) (2008) 854-866, Annual Meeting of the International-Societyof-Chemical-Ecology, Barcelona, SPAIN, 2006. doi:10.1007/s10886-0089484-5.

[2] J. MURLIS, J. ELKINTON, R. CARDE, ODOR PLUMES AND HOW INSECTS USE THEM, ANNUAL REVIEW OF ENTOMOLOGY 37 (1992) 505-532. doi:10.1146/annurev.en.37.010192.002445.

[3] A. Mafraneto, R. Carde, Effect of the fine-scale structure of phermone plumes - pulse frequency modulates activitation and upwind of almond moth males, Physiological Entomology 20 (3) (1995) 229-242. doi:10.1111/j.1365-3032.1995.tb00006.x.

[4] E. Vergassola, Massimo; Villermaux, B. I. Shraiman, 'infotaxis' as a strategy for searching without gradients, in: Nature, 2007, pp. 406-409.

[5] M. Dorigo, G. D. Caro, L. M. Gambardella, Ant algorithms for discrete optimization, Artificial Life (1999) 137-172.

[6] K. Diwold, A. Aderhold, A. Scheidler, M. Middendorf, Performance evaluation of artificial bee colony optimization and new selection schemes, Memetic Computing 3 (3) (2011) 149-162.

[7] E. Gelenbe, N. Schmajuk, J. Staddon, J. Reif, Autonomous search by robots and animals: A survey, Robotics and Autonomous Systems 22 (1) (1997) 23 - 34, biologically Inspired Autonomous Systems. 
doi:http://dx.doi.org/10.1016/S0921-8890(97)00014-6.

URL http://www.sciencedirect.com/science/article/pii/ S0921889097000146

[8] R. Russell, D. Thiel, R. Deveza, A. Mackay-Sim, A robotic system to locate hazardous chemical leaks, in: Robotics and Automation, 1995. Proceedings., 1995 IEEE International Conference on, Vol. 1, 1995, pp. 556-561 vol.1.

[9] W. Burgard, M. Moors, D. Fox, R. Simmons, S. Thrun, Collaborative multi-robot exploration, in: Robotics and Automation, 2000. Proceedings. ICRA '00. IEEE International Conference on, Vol. 1, 2000, pp. 476-481 vol.1. doi:10.1109/ROBOT.2000.844100.

[10] A. Marjovi, J. Nunes, L. Marques, A. de Almeida, Multi-robot exploration and fire searching, in: Intelligent Robots and Systems, 2009. IROS 2009. IEEE/RSJ International Conference on, 2009, pp. 19291934. doi:10.1109/IROS.2009.5354598.

[11] J. A. Farrell, S. Pang, W. Li, Plume mapping via hidden markov methods, Systems, Man, and Cybernetics, Part B: Cybernetics, IEEE Transactions on 33 (6) (2003) 850-863.

[12] A. Lilienthal, T. Duckett, Building gas concentration gridmaps with a mobile robot, Robotics and Autonomous Systems 48 (1) (2004) 3-15.

[13] G. Ferri, M. V. Jakuba, A. Mondini, V. Mattoli, B. Mazzolai, D. R. Yoerger, P. Dario, Mapping multiple gas/odor sources in an uncontrolled indoor environment using a bayesian occupancy grid mapping based method, Robotics and Autonomous Systems 59 (11) (2011) 988-1000.

[14] S. Kazadi, R. Goodman, D. Tsikata, H. Lin, An autonomous water vapor plume tracking robot using passive resistive polymer sensors, Autonomous Robots 9 (2) (2000) 175-188.

[15] G. Lytridis, Y. Virk, Rebour, E. Kadar, Odor-based navigational strategies for mobile agents, Adaptive Behavior 9 (3-4) (2001) 171-187.

[16] R. A. Russell, Robotic location of underground chemical sources, Robotica 22 (2004) 109-115. 
[17] R. A. Russell, A. Bab-Hadiashar, R. L. Shepherd, G. G. Wallace, A comparison of reactive robot chemotaxis algorithms, Robotics and $\mathrm{Au}-$ tonomous Systems 45 (2) (2003) 83-97.

[18] J. Farrell, S. Pang, W. Li, Chemical plume tracing via an autonomous underwater vehicle, Oceanic Engineering, IEEE Journal of 30 (2) (2005) 428-442. doi:10.1109/JOE.2004.838066.

[19] A. Lilienthal, T. Duckett, Experimental analysis of smelling braitenberg vehicles, Advanced Robotics 8 (8) (2004) 817-834.

[20] D. Chang, W. Wu, D. Webster, M. Weissburg, F. Zhang, A bio-inspired plume tracking algorithm for mobile sensing swarms in turbulent flow, in: Robotics and Automation (ICRA), 2013 IEEE International Conference on, 2013, pp. 921-926.

[21] H. C. Berg, E. M. Purcell, Physics of chemoreception, Biophysical Journal 20 (2) (1977) 193219.

[22] G. Falkovich, K. Gawȩdzki, M. Vergassola, Particles and fields in fluid turbulence, Rev. Mod. Phys. 73 (2001) 913-975.

[23] R. A. Russell, Locating underground chemical sources by tracking chemical gradients in 3 dimensions, in: Intelligent Robots and Systems, 2004. (IROS 2004). Proceedings. 2004 IEEE/RSJ International Conference on, Vol. 1, 2004, pp. 325-330 vol.1. doi:10.1109/IROS.2004.1389372.

[24] M. Eisenbach, Chemotaxis, Imperial College Press, London, 2004.

[25] D. Webster, M. J. Weissburg, The hydrodynamics of chemical cues among aquatic organisms, Annual Review of Fluid Mechanics 41 (2009) 73-90.

[26] W. Li, J. A. Farrell, S. Pang, R. M. Arrieta, Moth-inspired chemical plume tracing on an autonomous underwater vehicle, Robotics, IEEE Transactions on 22 (2) (2006) 292-307.

[27] P. Pyk, S. B. i. Badia, U. Bernardet, P. Knsel, M. Carlsson, J. Gu, E. Chanie, B. S. Hansson, T. C. Pearce, P. F. M. J. Verschure, An artificial moth: Chemical source localization using a robot based neuronal model of moth optomotor anemotactic search, Autonomous Robots 20 (3) (2006) 197-213. 
[28] N. Lemmens, S. de Jong, K. Tuyls, A. Nowe, Adaptive Agents and MultiAgent Systems III. Adaption and Multi-Agent Learning, ser. Lecture Notes in Computer Science, Vol. 4865, Springer, 2008, Ch. Bee behaviour in multi-agent systems, p. 145156.

[29] D. R. Webster, K. Y. Volyanskyy, M. J. Weissburg, Bioinspired algorithm for autonomous sensor-driven guidance in turbulent chemical plumes, Bioinspiration \& Biomimetics 7 (3) (2012) 036023.

URL http://stacks.iop.org/1748-3190/7/i=3/a=036023

[30] H. Ishida, T. Nakamoto, T. Moriizumi, Remote sensing of gas/odor source location and concentration distribution using mobile system, Sensors and Actuators B: Chemical 49 (1) (1998) 52-57.

[31] J.-B. Masson, Olfactory searches with limited space perception, Proceedings of the National Academy of Sciences 110 (28) (2013) 1126111266 .

[32] T. M. Cover, J. A. Thomas, Elements of Information Theory, WileyInterscience, New York, NY, USA, 1991. 